\title{
GIRO ESTÉTICO Y VANGUARDIA: ACERCA DE LA INFLUENCIA DEL PRAGMATISMO DE JOHN DEWEY EN ARTISTAS NORTEAMERICANOS
}

\author{
Aesthetic Turn and Vanguard: about the Influence of the Pragmatism of John \\ Dewey in American Artists
}

\section{Fabio Campeotto Claudio Marcelo Viale CONICET - Universidad Católica de Córdoba}

\begin{abstract}
Resumen: En la vasta producción del filósofo pragmatista John Dewey (1859-1952) la estética y la filosofía del arte ocupan un rol central a partir de la década del veinte, lo que puede denominarse su giro estético. El objetivo de este artículo, a través de un enfoque histórico-filosófico, es mostrar la influencia de este giro en el desarrollo de las artes en Estados Unidos. En la primera sección se exponen los tres ejes de la estética del pensador pragmatista que más han influido en los artistas norteamericanos luego de la publicación de Art as Experience (1934). En la segunda y tercera sección se investigan los vínculos entre las filosofías del arte y de la educación de Dewey y dos instituciones claves para el desarrollo del arte norteamericano: el Black Mountain College y el Federal Art Project. Finalmente, se muestra la centralidad del pragmatismo deweyano en las neovanguardias estadounidenses y la originalidad de su aporte en la definición del concepto de contracultura, tal como se desarrolló después de la Segunda Guerra Mundial.
\end{abstract}

Palabras claves: Pragmatismo, Federal Art Project, Black Mountain College, neovanguardias, educación, democracia.

\begin{abstract}
Within the extensive work of the pragmatist philosopher John Dewey (1859-1952), aesthetics and philosophy of art play a central role since the 1920 s, which can be called his "aesthetic turm". The aim of this article is to show, through an historical-philosophical approach, the influence of this turn in the development of the arts in the United States. In the first section we display the three central aspects of Dewey's aesthetics which have had a considerable influence among American artists after the publication of Art as Experience (1934). In the second and third section we investigate the links between Dewey's philosophies of art and education and two key institutions for the development of American art: The Black Mountain College and the Federal Art Project. Finally, we show the prominence of Dewey's pragmatism within American Neo-vanguards and the originality of his contribution in the definition of the concept of counterculture as it developed after the Second World War.
\end{abstract}

Keywords: Pragmatism, Black Mountain College, Federal Art Project, neo-vanguards education, democracy.

\section{Introducción}

Cuando John Dewey publica Art as Experience en 1934 era más conocido por su extensa trayectoria como pedagogo o filósofo de la educación que por sus teorías

\footnotetext{
${ }^{1}$ Las citas de Art as Experience (AE) pertenecen a la versión castellana traducida por J. CLARAMONTE. Barcelona: Paidós, 2008. Las referencias a otros textos de Dewey son de The Collected Works of John Dewey. J.A. BOYDSTON (ed.). Carbondale y Edwardsville: Southern Illinois University Press. Los textos se encuentran citados, tanto en el cuerpo del articulo como en las notas, según la siguiente subdivisión: $E W=$ Early Works; $M W=$ Middle Works; $L W=$ Later Works. Sus cartas se remiten a The Correspondence of John Dewey. $\mathrm{L}$. 
estéticas. En ese momento, ese etiquetamiento era cualquier cosa menos infundado, como lo muestra una mirada aunque sea superficial a su vasta producción. En otros términos: la estética y el arte no eran tópicos cruciales en sus escritos hasta ese momento, como Dewey mismo reconoce en una carta a su amigo Albert $\mathrm{C}$. Barnes ${ }^{2}$ en 1920: "Siempre he evitado la estética, el por qué no lo sé (...). Y ahora tengo una repulsión bastante firme en contra de toda la discusión estética". (Corresp. II, 04091)3.

A partir de la década del veinte, sin embargo, Dewey muestra un interés creciente en la estética, como puede apreciarse en su correspondencia y los textos que escribe a partir de entonces ${ }^{4}$. Una primera aproximación sistemática a estos tópicos es desarrollada en Experience and Nature (1925) culyo noveno capítulo se titula "Experience, Nature and $\operatorname{Art}^{\prime \prime}$ ( $L W, 1$, pp. 266-294). Esta incipiente estética presentada en Experience and Nature va desarrollándose hasta constituirse en uno de los ejes principales de su filosofía (casi una década más tarde) en Art as Experience, definido por Monroe Beardsley como "el trabajo sobre estética más importante escrito en inglés (y probablemente en cualquier idioma) (...) en nuestro siglo ${ }^{\circ 5}$.

El papel fundamental que la estética tiene en el pensamiento maduro de Dewey, en tanto, es motivo de numerosos análisis. Robert Westbrook, por ejemplo, sostiene que: "irónicamente, en el mismo momento en que Mumford lamentaba que en la filosofía de Dewey no había lugar para la estética, Dewey estaba empezando a poner el arte (...) en el

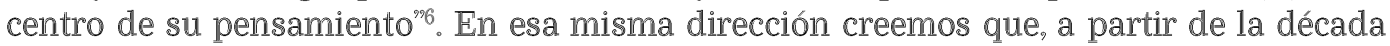
del veinte, existe un innegable giro estético en la filosofía de Dewey.

Sin embargo, a pesar de la estatura intelectual mundialmente reconocida de su autor y de la notable profundidad de Art as Experience, la reacción del ambiente filosófico tras la publicación del libro fue, en su mayoría, negativa?

A partir de la década del cincuenta, en tanto, Art as Experience corre el mismo derrotero que su autor: o bien padece un ostracismo similar al que sufre el pragmatismo clásico, o bien es objeto de críticas injustas y corrosivas originadas en el seno de una filosofía analítica que devino hegemónica. El comentario de Monroe Beardsley que citamos más arriba puede ser interpretado, entonces, como una voz aisladla dentro de un ambiente mayoritariamente hostil.

Contrastando, sin embargo, con el ostracismo y/o el rechazo que recibe su obra por parte del ambiente académico, tanto la filosofía de Dewey -en general- como Art as Experience -en particular- tuvieron una amplia difusión en los ambientes artísticos en

HICKMAN (ed.). Charlottesville: Intelex, 2008. Se citan particularmente cartas incluidas en el Volumen II (Corresp. II) y en el Volumen III (Corresp. III) Todas las citas de textos en lengua extranjera son traducciones nuestras, salvo que se indique lo contrario.

${ }^{2}$ Albert C. Barnes fue un empresario farmacéutico, coleccionista y crítico de arte de Filadelfia. Desde 1922 fue presidente de la Barnes Foundation, institución educativa de la cual Dewey fue director. Su influencia en la estética deweyana sólo recientemente ha ocupado un lugar en la literatura. A completar después de revisión.

${ }^{3}$ Dewey siempre tuvo un interés genuino en el arte (principalmente en la literatura) aunque el rol de la estética en su fillosofía no tuvo un desarrollo sistemático hasta los años veinte. Entre las distintas interpretaciones de la génesis de la estética deweyana creemos que la de Alexander Robins es la más relevante. Robins sostiene que existe una "estética fragmentadla" [piecemeal aesthetics] en la obra de Dewey antes de que sea desarrollada sistemáticamente a partir de la década del 20, gracias al papel decisivo que juegan A.C. Barnes y los intelectuales de su Fundación en lo que nosotros denominamos el giro estético de Dewey. ROBINS, Alexander. Aesthetics Experience and Art Appreciation: A Pragmatic Account. Tesis Doctoral en Filosofía. Universidad de Emory, Atlanta, 2015, pp. 15-17. A completar después de la revisión.

${ }^{4}$ En los años previos a la publicación de Art as Experience Dewey publica los ensayos "Individuality and Experience" y "Affective Thought" en 1925 ( $L W, 1$, pp. 1-6, 3-9). En 1926 publica "Art in Education-Education in Art" ( $L W, 2$, pp. 111-115). Finalmente, en 1931 escribe el ensayo "What Is It All About?" ( $L W, 6$, pp. 330-334).

${ }^{5}$ BEARDSLEY, Monroe. Aesthetics from Classical Greece to the Present: A Short History. Nueva York: The Macmillan Co., 1966, p. 332.

${ }^{6}$ WESTBROOK, Robert B. John Dewey and American Democracy. Ithaca y Londres: Comell University Press, 1991, p. 387. En la cita el autor se refiere al siguiente texto: MUMFORD, Lewis. The Golden Day: A Study in American Experience and Culture. Nueva York: Horace Liveright, 1926, p. 264.

${ }^{7}$ Véanse particularmente dos reseñas negativas: la primera es la de PEPPER, Stephen. "Some Questions on Dewey's Aesthetics". En SCHLIPP, Paul A., The Philosophy of John Dewey. Evanston: Northwestern University, 1939, pp. 369-390. La segunda es la de CROCE, Benedetto. "Intomo all'estetica del Dewey". En La Critica, 38, 1940, pp. 348-353. 
un período que se extiende desde la década del treinta hasta, por lo menos, los años '70. La paradójica situación ha sido inequívocamente descripta por Yoann Barbereau:

Los años que vieron la estética analítica dominar el debate filosófico son también aquellos donde, en la escena artística, se recita una pieza, cuyo centro está ocupado por quien el teatro de las operaciones filosóficas había empujado fuera del escenario: Art as Experience ${ }^{8}$.

Esta influencia directa del giro estético de Dewey en prominentes artistas modernos es un tópico frecuentemente descuidado en la literatura. Si bien dos recientes contribuciones de Ramón del Castillo y Mary Jane Jacob ${ }^{10}$ tratan tangencialmente con este tema, existen solo unos pocos trabajos escritos en inglés ${ }^{11}$, en francés ${ }^{12}$ y en italiano ${ }^{13}$ que pretenden operar una reconstrucción sistemática de la efectiva influencia de Dewey en el desarrollo de prácticas artísticas.

En ese marco, este artículo se propone recortar, reconstruir y analizar algunas características fundamentales de la filosofía de Dewey que más han influido en el desarrollo de dos instituciones creadas entre las dos Guerras Mundiales (el Black Mountain College y el Federal Art Project) con el objetivo de rehabilitar el nombre de Dewey no solo en el campo de la filosofía y de la crítica artística (propósito que Jacob alcanza eficazmente en su libro) sino también en el de la historia del arte. Para ello se articula el trabajo en cuatro partes. En la primera (Art as Experience: tres tópicos fundamentales) se exponen ejes claves de su pensamiento estético que han sido los más influyentes en los artistas norteamericanos a partir de la década del treinta. En la segunda y tercera sección (Democracia, educación y arte: Dewey y el Black Mountain College, El arte en tiempos de Gran Depresión: Dewey y el Federal Art Project) se analiza la relevancia trascendental del pensamiento de Dewey (de su estética principalmente, pero también de su pedagogía y su filosofía política) en el desarrollo de esas dos instituciones que subvencionaron a más de una generación de artistas. En la cuarta sección (John Dewey: pragmatismo y arte) se muestran las características centrales de la influencia de $\mathbb{D}$ ewey en artistas norteamericanos de las llamadas neovanguardias ${ }^{14}$. Finalmente, se establecen unas conclusiones.

\section{Art as Experience: tres ejes fundamentales}

\footnotetext{
8 BARBEREAU, Yoann. "Expérience et performance. Fragments d'un dialogue pragmatiste". En Revue d'Esthétique, 44, 2003, p. 25.

${ }^{9}$ DEL CASTILLO, Ramón. "La corrosión de la experiencia. Populismo, abstracción y cultura de masas. En ARENAS, L., FAERNA, M., DEL CASTILLO, R., John Dewey. Una estética de este mundo. Zaragoza: Prensas de la Universidad de Zaragoza, 2018, pp. 77-96.

${ }^{10}$ JACOB, Mary Jane. Dewey for Artists. Chicago y Londres: The University of Chicago Press, 2018.

${ }^{11}$ BUETTNER, Stewart. "John Dewey and the Visual Arts in America". En Joumal of Aesthetics and Ant Criticism, 33, 1975, pp. 383-391; BERUBE, Maurice R. "John Dewey and the Abstract Expressionists". En Educational Theory, 48, 1998, pp. 211-227; JAY, Martin. "Somaesthetics and Democracy: Dewey and Contemporary Body Art". En Journal of Aesthetic Education, 36, 2002, pp. 55-69; DREON, Roberta. "Was Art as Experience Socially Effective? Dewey, the Federal Art Project and Abstract Expressionism". En The European Journal of Pragmatism, 5, 2013, pp. 279-292.

${ }^{12}$ BARBEREAU, Yoann. Ob. Cit:; PIRÓN. Francois. "Se rire de l'échec: le pragmatisme de George Maciunas". En Revue Initiales ,1, 2009, pp. 27-32; WIAME, Aline. "L'art comme expérience et la pragmatique du spectateur: entre performance et philosophie". En Tangence, 108, 2015, pp. 13-27.

${ }^{13}$ SENALDI, Marco. "Art as Experience e l'arte contemporanea". En RUSSO, L., Esperienza estetica a partire da John Dewey. Palermo: Centro Internazionale Studi di Estetica, 2007, pp.49-60.

${ }^{14}$ Si bien este artículo se limita al panorama estadounidense, es sabido que la filosofía de Dewey ha influido también en el desarrollo del arte afuera de Estados Unidos. En la segunda sección de este artículo, por ejemplo, se hace referencia a la Bauhaus alemana. Además, existen conexiones, aún poco exploradas, entre la estética de Dewey y el movimiento "Arte Povera", que se desarrolla en Italia en los años '60. MANGINI, Elizabeth. "Feeling One's Way through a Cultural Chiasm: Touch in Giuseppe Penone Sculpture c. 1969". En PARATI, Graziella, New Perspectives in Italian Studies. Vol 2: The Arts and History. Madison y Teaneck: Farleigh Dickinson University Press, 2012, pp. 153-174. El texto de Renato Barilli Per un'estetica mondana (Boloña: Il Mulino, 1964) ha sido un estímulo para una recepción positiva de las ideas deweyanas en el ambiente cultural italiano. Se agradece a Roberta Dreon por habernos sugerido este dato.
} 
Cualquier aproximación a Art as Experience debe necesariamente asumir como punto de partida dos factores peculiares. Por un lado, el libro es la culminación de un proceso (su giro estético) que se produce en la filosofía de Dewey a partir de la década del '20, cuando se encontraba en su plena madurez (había cumplido 60 años). Por el otro, Art as Experience sobresale dentro de la inmensa producción del gran pragmatista clásico, como un texto crucial para entender no sólo su pensamiento estético, sino toda su filosofía madura. Thomas M. Alexander subraya este punto con gran claridad:

Dewey no se acercó al tema de la experiencia estética (...) simplemente para "completar" su fillosofía. Dewey afirma explícitamente en el libro que la prueba para averiguar la capacidad de entender la experiencia se encuentra en la dimensión estética (...) en este sentido Art as Experience debe ser interpretado como un texto central y crucial. La experiencia para Dewey se comprende más plenamente desde el punto de vista del arte. El arte muestira que la experiencia puede ser inteligente y creativamente interiorizada y transformada ${ }^{15}$.

En esta sección se analizan los tres ejes de la estética deweyana que más han influido en el desarrollo del arte estadounidense a partir de los años treinta: su concepción de experiencia estética, en primer lugar, la crítica que Dewey hace de los lugares destinados al goce estético (museos, galerías, etc.) en segundo lugar y finalmente, el rol que el filósofo concibe para el espectador del arte.

Se puede empezar a entender la concepción de experiencia estética de Dewey (primer eje) a partir de una distinción de Roberta Dreon. En su notable libro Fuori dalla torre d'avorio, Dreon sostiene que la estética de Dewey posee un indudable carácter "inclusivo", que la diferencia notablemente de la tendencia "exclusiva" de buena parte de la tradición moderna.

Las características básicas que distinguen este conjunto de reflexiones son, por lo menos, dos. Conciernen, por un lado, la extensión explícita de los aspectos estéticos a cualidades que incluyen a la experiencia en cuanto tal. Por el otro, se perfila netamente la extensión de lo artístico mucho más allá del ámbito elitista de las llamadas bellas artes o Artes altas, sino también más allá de las artesanías muy a menudo contrapuestas a ellas, a todas las prácticas vitales humanas ${ }^{16}$.

La primera cuestión que Dreon señala, "la extensión (...) de los aspectos estéticos a cualidades que incluyen la experiencia en cuanto tal", gira alrededor del rol imprescindible que juega la experiencia en todas las actividades humanas y, entre ellas, el goce estético. Un primer punto a analizar es lo que Dewey entiende por experiencia estética y su estrecha relación con la experiencia ordinaria. Según la definición del autor, la experiencia estética es "la experiencia libre de las fuerzas que impiden y confunden su desarrollo como experiencia; es decir, libre de factores que subordinan la experiencia tal como se tiene, a algo que está más allá de ella misma" ( $A E$, p. 309). En el primer capítulo de Art as Experience, titulado "La criatura viviente", Dewey subraya reiteradamente la relación entre la experiencia estética y la experiencia ordinaria.

Afortunadamente, una teoría del lugar de lo estético en la experiencia no tiene que perderse en detalles minúsculos cuando parte de la experiencia en su forma elemental, porque bastan solo amplios lineamientos. La primera gran consideración es la de que la vida se produce en un ambiente: no solamente en este, sino a causa de éste, a través de una interacción con el mismo. Ninguna criatura vive meramente bajo su piel; suis órganos subcutáneos son medios de

${ }^{15}$ ALEXANDER, Thomas M. John Dewey's Theory of Art, Experience and Nature: The Horizons of Feeling. Albany: Suny Press, 1987, pp. 184-185.

${ }^{16}$ DREON, Roberta. Fuori dalla torre d'avorio: I'estetica inclusiva di John Dewey oggi Génova y Milán: Marietti, 2012, pp. 7-8. 
conexión con lo que está más allá de su constitución corpórea y con lo que debe ajustarse, a fin de vivirir, por acomodación y defensa y también por conquista ( $A E_{\text {, }}$ pp. 14-15).

Este último párrafo es de gran importancia porque muestra dos ejes cruciales de la concepción deweyana de experiencia: que la experiencia estética se desarrolla a partir de una "experiencia en su forma elemental", en primer lugar y que no hay experiencia sin la interacción del individuo con su entorno, en segundo lugar. Más precisamente, para Dewey "la experiencia es el resultado, el signo y la recompensa de esta interacción del organismo y el ambiente, que cuando se realiza plenamente es una transformación de la interacción en participación y comunicación" ( $A E$, p. 26).

Lo que distingue una experiencia como estética, en tanto, es su carácter consumatorio, es decir, cuando la experiencia es "la conversión de la resistencia y la tensión, de las excitaciones que tientan a la distracción, con un movimiento hacia un final satisfactorio e inclusivo" ( $A E$, p. 64). En la experiencia estética "un cuerpo de materias y significados no estéticos por sí mismos, se hacen estéticos cuando tornan un movimiento ordenado y rítmico hacia su consumación" ( $A E$, p. 369).

Este carácter consumatorio de la experiencia estética tiene que tener como punto de partida la experiencia ordinaria. Para esto, el concepto deweyano de rodeo [detour] juega un rol central ${ }^{17}$.

Al fin de entender la significación de los productos artísticos, tendremos que olvidarlos por el momento y hacerlos a un lado, para recurrir a las fuerzas y condiciones ordinarias de la experiencia que no acostumbramos a considerar como estética. Tenemos que Ilegar a la teoría del arte por medio de un rodeo [detour](...). A fin de entender lo estético en sus formas últimas y aprobadas, se debe empezar con su materia prima; con los acontecimientos y escenas que atraen la atención del ojo y del oído del hombre despertando su interés y proporcionándole goce mientras mira y escucha (AE, pp.4-5. Cursiva nuestra).

Más adelante en el texto el filósofo retoma nuevamente el concepto de detour, que describe como una operación indispensable para una plena comprensión del arte.

La comprensión del arte y de su papel en la civilización, no resulta faṽorecida con elogios, ni ocupándose exclusivamente en grandes obras de arte reconocidas como tales. La comprensión que intenta la teoría será lograda mediante un rodeo Idetourj: regresando a la experiencia de lo común o rondando las cosas para descubrir la cualidad estética que tal experiencia posee. La teoría puede partir de reconocidas obras de arte solamente cuando se supone que la estética es uin compartimento, o solamente cuando las obras de arte se colocan en un nicho aparte en vez de ser celebraciones de las cosas de la experiencia ordinaria, reconocidas como tales ( $A E_{\text {, }}$ pp. 11-12. Cursiva nuestra).

El concepto de detour resulta crucial también para entender el segundo aspecto mencionado por Dreon, "Ia extensión de lo artístico mucho más allá del ámbito elitista de las llamadas bellas artes o Artes altas (...) a todas las prácticas vitales humanas".

Las ideas que colocan el arte en un pedestal remoto, penetran tan sutilmente y están tan extendidas, que muchas personas sentirán repugnancia más bien que agrado, si se les dice que gozan en sus acostumbradas recreaciones, en parte al menos, por su cualidad estética. Las artes que hoy tienen mayor vitalidad para el hombre de a pie son cosas que no considera como arte; por ejemplo, el cine, el jazz, firecuentemente la página cómica, los relatos periodísticos de amores, asesinatos y correrías de bandidos (AE, p. 6).

${ }^{17}$ A completar después de la evaluación. 
Esta extensión de la estética a las diversas prácticas cotidianas es uno de los aportes más novedosos de Dewey a la teoría artística contemporánea ya que abre el camino a una definición de lo artístico que trasciende los límites convencionales impuestos por la cultura occidental. En términos de Dreon:

[R]econocer la naturalidad de las prácticas artísticas en las formas de vida humana más diversas no signifíca caer en una versión de ahistoricismo ingenuo, y tampoco de conservadurismo político. Significa, más bien, reconocer que la concepción del arte del siglo XIXX - (...) como contemplación desinteresada y autónoma con respeto a otras esferas de valores, así como carente de conocimiento- es fuertemente etnocéntrica y corta de miras sobre el plano ético y socia $1^{18}$.

El segundo eje de la estética de Dewey que se presenta gira alrededor de su visión de museo y de las galerías de arte ${ }^{19}$. Sus críticas se dirigen en particular al rol de estas instituciones culturales que tradicionalmente se han ocupado de promover el goce estético:

(..o) cuando lo que [el hombre] conoce como arte se relega al museo o a la galería, el incontenible impulso hacia experiencias que se pueden gozar en sí mismas encuentira tantos escapes cuantos el ambiente provee. Muchas personas que protestan contra la concepción del arte como objeto de museo, sin embargo, comparten la falacia de la que brota aquella concepción. La noción popular proviene de una separación del arte de los objetos y escenas de la experiencia ordinaria, que muchos teóricos se jactan de sostener y elaborar. Ein el momento en que los objetos selectos y distinguidos se conectan estrechamente con el producto de vocaciones usuales, es cuando la apreciación de aquéllos es más maduira y más profunda. Cuando determinados objetos son reconocidos como obras de arte por la gente cultivada, se revelan insustanciales a la masa del pueblo por su lejanía; es en este momento en el que el hambre estética está dispuesta a buscar lo barato y lo vulgar ( $A E_{,}$, p. 6).

Para Dewey la gran mayoría de los museos de Europa, o bien son "conmemoraciones del ascenso del imperialismo y el nacionalismo" ( $A E$, p. 9), o bien son el producto del capitalismo moderno, con el capitalista que "para evidenciar su buena posición en el mundo de la alta cultura, amontona pinturas, estatuas, joyas artísticas, así como su caudal y sus bonos acreditan su situación en el mundo económico" (AE, p. 9). En ambos casos, las instituciones culturales promueven un goce de objetos que

reflejan y establecen un estado cultural superior, pero su segregación de la vida común refleja el hecho de que no son parte de una cultura nativa y espontánea [.... ]. Como obras de arte han perdido su estado vernácuilo y han adquirido uno nuevo, que es el de ser ejemplares de las bellas artes, y nada más. Además, las obras de arte se producen ahora, como otros artículos, para seir vendidas en el mercado" ( $A \bar{E}$, pp. 9-10).

La crítica de Dewey a las instituciones culturales apunta principalmente a hacer visibles dos falacias estructurales. En primer lugar, la total desconexión de los museos con la vida cotidiana y su tendencia a alejar el arte de la experiencia ordinaria. En segundo lugar, el goce meramente pasivo del arte que museos y galerías promueven. La sedimentación de estas costumbres estéticas en la sociedad se encuentra, otra vez, vinculada con las teorías trascendentes que remiten el arte a un reino separado y lo alejan definitivamente de la vida común.

Hay que indicar que las teorías que aíslan el arte y su apreciación colocándolos

${ }^{18}$ DREON, Roberta. Fuori dalla torre d'avorio, Ob. Cit., p. 15

${ }^{19}$ A completar después de la evaluación. 
en un reino que les es propio, desconectados de otros modos de experiencia, no son incuestionables al tema, sino que aparecen a causa de condiciones específicas extrañas. Como estas condiciones están incluidas en instituciones y costumbres de vida, operan de modo efectivo porque su acción es inconsciente. Entonces, el teórico presuime que están incluidas en la naturaleza de las cosas. Sin embargo, la influencia de estas condiciones no está confinada a la teoría. Como he indicado ya, afecta profundamente la práctica de la vida, sustrayendo las percepciones estéticas, que son ingredientes necesarios de la felicidad, o las reducen a excitaciones de compensación, agradables y pasajeras ( $A \bar{H}, \underline{p} .11$ ).

Este último punto -la crítica a la percepción pasiva de objetos artísticos- refiere al tercer eje de Art as Experience que se analiza en esta sección, el rol que Dewey cree que debería tener el espectador del arte. Dewey se opone, como se ha visto, a la concepción de obra de arte como producto acabado y apto a un disfrute meramente pasivo. Para el filósofo norteamericano la obra de arte es más bien el resultado de un proceso, en el cual el artista productor está involucrado activa y dinámicamente con su entorno.

El arte está, pues, prefigurado en cada proceso de la vida. Ūn pájaro construye su nido y un castor su casa, cuando las presiones orgánicas internas cooperan con los materiales externos de manera que las primeras se cumplan y los últimos se transformen en una culminación satisfactoria. Podríamos dudar de aplicar la palabra arte, si dudamos de la presencia de una intención directiva. No obstante, toda deliberación, todo intento consciente nace de cosas ejecutadas orgánicamente a través de un juego mutuo de energías naturales. Si no fuera así, el arte estaría construido sobre arena movediza o, aún más, sobre el aire inestable ( $A \bar{F}$, p. 28).

Dewey hace un uso frecuente de términos como "proceso", "acción", "energía", "movimiento" etc., para subrayar la actividad propia no sólo del artista creador sino también del espectador que goza del arte. En sus propias palabras: "[h]] subrayado el hecho de que toda experiencia integral se mueve hacia un término, un fin, ya que cesa solamente cuando sus energías activas han hecho su propia labor. Esta clausura de un circuito de energía es lo opuesto a la suspensión, a la stasis" ( $A E_{,}, p_{.} 47$. Cursiva nuestra). Sobre la acción que caracteriza el proceso de producción artística Dewey afirma lo siguiente:

El arte denota un proceso de hacer o elaborar. Esto es cierto tanto para las bellas artes como para el arte tecnológico. El arte comprende modelar el barro, esculpir el mármol, colar el bronce, aplicar pigmentos, construir edificios, cantar canciones, tocar instrumentos, representar papeles en el escenario, realizar movimientos rítmicos en la danza. Cada arte hace algo con algún material físico, el cuerpo o algo fuera del cuerpo, con o sin el uso de herramientas, y con la mira de producir algo visible, audible o tangible. Tan marcada es la fase activa del arte que los diccionarios lo definen usualmente en términos de acción diestra, de habilidad en la ejecución (AE, p. 54. Cursiva nuestra).

La acción, sin embargo, no es exclusiva del productor. Dewey no acepta papeles pasivos: si la experiencia estética se caracteriza por su organización dínámica, el espectador también debe actuar dinámicamente en ella.

No es tan fácil en el caso del espectador, entender la unión íntima entre el hacer y el padecer, como en el caso del productor. Solemos suponer que el espectador asimilla tan sólo lo que está concluido, y no advertimos que este asimilar irmplica actividades comparables a las del creador. Sin embargo, receptividad no es pasividad. Es también un proceso que consiste en una serie de actos de respuesta que se acumulan, hasta llegara la satisfacción objetiva ( $A E_{q}$ p. 60). 
El espectador, por tanto, debe pasar por su propia experiencia estética; en otras palabras, debe vivir un proceso que, por cualidad e intensidad, es asimilable al del creador. En términos de Dewey: "Para percibir, un contemplador debe crear su propia experiencia. Y esta creación debe incluir relaciones comparables a las que sintió el creador (...). El artista selecciona, simplifica, aclara, abrevia y condensa de acuerdo con su interés; y el contemplador debe pasar estas operaciones, de acuerdo con su punto de vista y su interés" ( $A E$, p. 62).

\section{Democracia, educación y arte: Dewey y el Black Mountain College}

Aún antes de la publicación de Art as Experience, John Dewey no era un pensador desconocido en el ambiente artístico internacional. Tenía, por ejemplo, un vínculo de amistad y estima mutua con el artista francés Henri Matisse, desarrollado durante la estadía del pintor en Estados Unidos a principios de los años '30 y que continúa a través de un intercambio epistolar ${ }^{20}$.

Es sabido, además, que los trabajos de Dewey sobre educación encontraron una amplia difusión en los ambientes de la Bauhaus en Alemania. Margaret Katgens - Craig, en su libro The Bauhaus and America, analiza la innegable influencia del pragmatismo sobre la concepción educativa de la Bauhaus:

Su curriculum experimental y orientado hacia la práctica acercó la Bauhaus al pragmatismo norteamericano. Aprender haciendo [leaming by doing] (...), la educación del individuo para el bien de la comunidad y los objetivos de la escuela, retoman la concepción de John Dewey (...). Varios artistas que trabajaban en la Bauhaus habían establecido contactos con Estados Unidos ya antes de 1919. Lectores, docentes, estudiantes y visitantes estadounidenses no eran raros en la Bauhaus a finales de los años ${ }^{6} 20^{21}$.

Democracy and Education, cuya traducción se publica en alemán en 1916, influye en muchos artistas alemanes en general y en Josef Albers en particular ${ }^{22}$. El pintor oriundo de Westfalia, emigrado a Estados Unidos en 1933, leyó Art as Experience tras su publicación y escribió dos breves artículos inspirados por el pensamiento de Dewey ${ }^{23}$. Más adelante se retoma la conexión entre Dewey y la Bauhaus.

Fueron dos instituciones norteamericanas, en tanto, el Black Mountain College (BMC, 1933-1957) y el Federal Art Project (FAP, 1935-1943) las que intentaron impulsar las ideas de Dewey sobre educación y estética y promover su aplicación práctica en el campo de las artes. $\mathrm{El} B M C$, que pasa a la historia principalmente como un laboratorio de experimentación artística de vanguardia, fue fundado en 1933 en Carolina del Norte por el partidario de la educación progresiva (y lector entusiasta de Dewey) John Andrew Rice, procedente del Rollins College en Winter Park, Florida. Rice fue acompañado desde la fundación del BMC hasta 1949 por Theodore Dreier, él también ex profesor del Rollins y amigo de larga data de la familia de Dewey.

Si bien el BMC fue fundado en circunstancias particulares (los primeros profesores y estudiantes eran disidentes del Rollins College, siempre vivió una situación económica precaria y a menudo encontró el ostracismo tanto de la comunidad local como de los ambientes más conservadores de la sociedad estadounidense) en sus apenas 23 años de vida se impuso como una de las instituciones educativas más importantes

\footnotetext{
${ }^{20}$ UENO, Masamichi. Democratic Education and the Public Sphere: Towards John Dewey's Theory of Aesthetic Experience. Nueva York: Routledge, 2016, pp. 129-132

${ }^{21}$ KATGENS-CRAIG, Margaret. The Bawhaus and America: First Contacts (1919-1936). Cambridge y Londres: MIT Press, 1999, p. 212.

${ }^{22}$ DÍAZ, Eva. The Experimenters: Chance and Design at Black Mountain College. Chicago y Londres: University of Chicago Press, 2015, p. 46.

${ }^{23}$ ALBERS, Josef. "Art as Experience". En Progressive Education, 12, 6, 1935, pp. 391-393;" "A Note on the Arts in Education". En American Magazine of Art, 29, 4, 1936, p. 233.
} 
para el desarrollo de las artes en la historia de Estados Unidos. El legado dejado por el college va entonces mucho más allá de la prestigiosa presencia de Dewey en su consejo consultivo. Eva Díaz así describe el inmenso patrimonio que el BMC ha dejado en la cultura norteamericana:

Poco después de la Segunda Guerra Mundial, en el Black Mountain College la experimentación fue adoptada por algunas facultades y estudiantes que rechazaban la deriva hacia el Expresionismo. Se puede también llamar experimentación, en toda su diversidad, la idea misma del Black Mountain College, que ha marcado un momento importante, y aun relativamente ignorado en la historia de las prácticas artísticas, de la pedagogía, y del modernismo en general en la historia norteamericana de mitad del siglo. Lo que se destaca de este momento no fue una única alternativa al Expresionismo. Los modelos experimentales que proponían Josef Albers, John Cage y Buckminster Fuller [...] representain un conjuinto de distintas direcciones tomadas por el arte, la música y la arquitectura después de la Guerra ${ }^{24}$.

Entre los docentes que enseñaron en la escuela se encuentran prestigiosos artistas estadounidenses, como los pintores Franz Kline, Willem de Kooning y Robert Motherwell, el músico John Cage, el bailarín Merce Cunningham, el escultor Robert Rauschenberg, el arquitecto Buckminster Fuller y el poeta Charles Olson. El BMC fue además el refugio de exiliados alemanes procedentes del movimiento Bauhaus, llegados a Estados Unidos luego de que el régimen Nazi cerró la famosa escuela en 1933. Además del ya mencionado Josef Albers y de su esposa Anni, también el mismo fundador de la Baulhaus, Walter Gropius, transitó por el BMC. Entre los conferencistas más ilustres se recuerdan Albert Einstein y los escritores William Carlos Williams y Mary Caroline Richards. Henry Miller, Aldous Huxley y Allen Ginsberg visitaron el college, mientras que Robert De Niro Sr. (artista plástico y padre del conocido actor), Kenneth Noland (pintor), Ruth Asawa (escultora), Robert Creeley, Ed Dorn (poetas) y Jonathan Williams (escritor) fueron sus reconocidos estudiantes.

Si bien la presencia de Dewey en la institución fue, más que nada, nominal (Dewey visitó el college ocasionalmente, aunque mantuvo contactos epistolares con Rice y Dreier $)^{25}$, sus ideas manifiestamente se reflejaban en la organización de la escuela, en su curriculum y en las líneas guías que desde su fundación han acompañado y marcado su desarrollo. Este dominio de la filosofía deweyana dentro de la institución ha sido subrayado, entre otros, por Jonathan Fisher:

El BMC fue único entre otras instituciones "progresivas" de educación superior en cuanto recibió la clara aprobación del mismo Dewey. Esto no quiere decir que el pragmatismo de Dewey fuera el único conjunto de ideas en funcionamiento en la comunidad del BMC, ni debe implicar que Dewey nunca aprobó otras universidades o currículos universitarios. Sin embargo, debido a su dominio como conjunto de principios rectores durante los años '30 y ' 40 , la filosofía progresiva de Dewey proporcionaba un sustrato sobre el cual las otras ideas, estructuras y procesos del BMC deben ser examinados ${ }^{26}$

La influencia del gran pragmatista clásico (particularmente de sus ideas expuestas en Democracy and Education) es evidente si se toman en consideración las

\footnotetext{
${ }^{24}$ DÍAZ, Eva. Ob. Cit., p. 149.

${ }^{25}$ Dewey visitó el BMC en 1935-36. Durante sus estadías Dewey no participó activamente en la vida académica ni influyó en el desarrollo de la actividad académica, se limitó a asistir a clases. Su presencia en el consejo consultivo era una fuente de prestigio para la siempre difícil recaudación de fondos. REYNOLDS, Katherine C. "Progressive Ideals and Experimental Higher Education: The Example of John Dewey and Black Mountain College". En Education and Culture, 14, 1, 1997, p. 6.

${ }^{26}$ FISHER, Jonathan. The Life and Work of the College: Towards the History of Black Mountain College as an Institution of Progressive Higher Education Tesis para optar al grado de Magíster en Educación, Universidad de British Columbia, Vancouver, 2013, pp. 7-8.
} 
tres líneas guías de la escuela: la prevalencia de la enseñanza práctica sobre la asimilación pasiva de contenidos teóricos, en primer lugar; la libertad que el estudiante tenía en elegir su propio trayecto de formación, en segundo lugar y finalmente, la voluntad de crear una institución que no sea simplemente una escuela, sino una comunidad basada en los principios de democracia y convivencia.

El primer aspecto ha sido tratado ampliamente por Dewey en sus teorías pedagógicas. En Democracy and Education, por ejemplo, se lee:

El estudio de la vida mental ha evidenciado el valor fundamental de las tendencias innatas a la exploración, a la manipulación de herramientas y materiales, a la construcción, a sentir una emoción jubilosa, etc. Cuando ejercicios estimulados por estos instintos son parte integral del programa escolar, el alumno está totalmente involucrado, se reduce la brecha artificial entre la vida en la escuela y la vida afuera, se brindan motivos para prestar atención a una gran variedad de materiales y procesos clara y realmente educativos, y se proporcionan asociaciones cooperativas que brindan información a un entorno social. En resumen, los motivos para asignar al juego y al trabajo activo un lugar definido en el plan de estudios son intelectuales y sociales, no son cuestiones de conveniencia temporal y de complacencia momentánea. Sin algo así, no es posible asegurar el estado normal del aprendizaje ( $M W, 9, p .203)$.

La centralidad de las disciplinas artísticas en la oferta formativa del BMC era, en efecto, funcional al desarrollo de dos aspectos: la intensificación del componente experiencial en el aprendizaje de los alumnos, por un lado; la estimulación de su poder imaginativo, por el otro. En términos de Catherine Reynolds:

Para Dewey, estas ideas fueron estrechamente relacionadas con su compromiso con la democracia en la educación; la práctica del arte y otros trabajos manuales debían ser incluidos porque al menos algunos estudiantes podrían tener mayor interés o habilidad en ellos. Rice y Albers no estaban en desacuerdo, pero fueron más allá: la práctica del arte debía ser emprendida por todos los estudiantes- ya sean economistas en ciernes, futuros periodistas o grabadores prometedorespara inculcar hábitos de disciplina y observación ${ }^{27}$ (Cuirsiva nuestra).

La prevalencia de las llamadas artes liberales en el plan de estudio del college ha sido "pragmáticamente" justificada por Rice:

Casi cada hombre es un poco un artista, por lo menos potencialimente es una persona con imaginación, que puede ser desarrollada; $\mathrm{y}$, hasta donde sé, hay solo una forma para entrenarlo y desarrollarlo - la forma que no descubrí yo sino el Black Mountain College en conjuinto. Aquíi nuestiro esfuerzo central y consecuente es de enseñar métodos, no contenidos; de enfatizar procesos, no resultados; de sugerir al estudiante en entender que la forma de gestionar a los hechos y a sí mismo en los hechos es más importante que los hechos de por $s i^{28}$.

El segundo eje del $\mathrm{BMC}$, en tanto, es la libertad que las autoridades escolares dejaban al estudiante en la organización de su propio curriculum. En otras palabras, el BMC defendía los intereses y las aptitudes del estudiante en su individualidad. Sobre este tema Dewey escribe:

La individualidad como factor a respetar en la educación tiene un doble significado. En primer lugar, uno es mentalmente un individuo solo porque tiene su propio propósito y problema, y lleva a cabo su propio pensamiento (...). En

\footnotetext{
${ }^{27}$ REYNOLDS, Catherine. Ob. Cit p. p. 5.

${ }^{28}$ En ADAMIC, Louis. "Education on a Mountain: The Story of Black Mountain College". En Harper's Monthly Magazine, 172, 1936, p. 518.
} 
segundo lugar, de persona a persona hay variaciones de punto de vista, de forma de tratar los objetos y de modo de abordaje. Cuando estas variaciones se suprimen en nombre de supuestos intereses de la uniformidad, y se intenta tener un único molde de método de estudio y lectura, resulta inevitable la confusión mental y la artificicialidad. La originalidad se destruye gradualmente, se socava la confianza en la propia calidad de la operación mental y se inculca un sometimiento dócil a la opinión de los demás, o las ideas están fuera de control [run will] $(\mathbb{M W}, 9,1.313)$.

El curriculum del BMC estaba formado por solo dos cursos obligatorios: "Platón I", dictado por Rice, y un curso introductorio a los materiales y a las formas del arte, dictado por Albers. En su carrera, por tanto, el alumno era libre de elegir entre distintas disciplinas, no estrictamente artísticas, con el auxilio de un tutor que lo ayudaba a organizar su propio plan personal.

Aunque era un experto en clásicos, Rice redujo su relevancia a una de muchas opciones. Era especialmente crítico con el sistema tradicional de certificación y credencialización porque un plan de estudios uniforme, juntos con un énfasis en la acumulación de conocimiento, generaba un vocabulario, culto pero irrelevante, de formas vacías. En su lugar, favoreció un diálogo socrático, conectando preguntas existenciales con el desarrollo del estudiante y haciendo hincapié en el aprendizaje sobre la base de la investigación y el estudio. Rice no aceptaba ninguna regla de expresión oral y escrita. Era importante para él (...) que los estudiantes no fueran forzados dentro de un sistema fijo o una torre de marfiil ${ }^{29}$.

El tercer eje relativo a la organización del BMC gira alrededor de la idea de comunidad, fundada y regida democráticamente. Karl-Heinz Füssl observa:

Además de la coeducación, se establecieron nuevas estructuras de autogobierno para reducir el grado de relación estrictamente jerárquica entre profesores y alumnos. Los habituales consejos de adiministración, decanatos y presidencia fueron reemplazados por un consejo consultivo y un rectorado rotativo. Un consejo electo entre los estudiantes y la facultad aprobaba todas las decisiones importantes ${ }^{30}$.

El catedrático de Yale Charles Perrow, ex estudiante del college entre 1946 y 1948 , describe el clima de revolución cultural y participación democrática que se vivía en la institución y que ella misma estimulaba en sus integrantes:

Con el fin de la Guerra y del Fascismo, el college intensificó el impulso norteamericano hacia la democracia, el estallido cultural y la revolución sexual (...). El Black Mountain era seguramente un holograma de la preocupación pendiente de la nación con el gobiemo democrático, las innovaciones culturales y la libertad sexual ${ }^{31}$.

Dewey, a lo largo de toda su carrera de pedagogo, no sólo defendió conceptualmente la idea de la democracia en la educación, sino también estimuló la construcción de la democracia a través de la educación ${ }^{32}$. En una carta enviada a Theodore Dreier en 1940 Dewey subraya este aspecto crucial:

\footnotetext{
${ }^{29}$ FÜSSL, Karl-Heinz. "Pestalozzi in Dewey's Realm? Bauhaus Master Josef Albers among the German- speaking Emigrés' Colony at Black Mountain College (1933-1949)". En Pedagogica Historica, 42, 1-2, 2006, p. 82.

${ }^{30} \mathrm{Idem}$, Ibidem, p. 78.

${ }^{31}$ PERROW, Charles. "Drinking Deep at Black Mountain College". Ein Southern Cultures, 19, 4, 2013, p. 80.

${ }^{32}$ Sobre este punto, el gran pragmatista clásico afirma en Democracy and Education: "No es accidental que todas las democracias hayan puesto una gran atención sobre la educación; que la educación haya sido su primer cuidado y su problema persistente. Solo a través de la educación la igualdad de oportunidades puede 
Espero, fervientemente, que sus esfuerzos para obtener el apoyo adecuado para el Black Mountain College tengan éxito. El trabajo y la vida del College (es imposible en este caso separar los dos) es uin vivo ejemplo de democracia en acción. A pesar de cómo se desarrolle la crisis actual, el tipo de trabajo que el College hace es una necesidad imperativa para los intereses de la demociacia a largo plazo. $\mathbb{E} 1$ College existe como 'base' para una forma democrática de vida (Corresp. III, 13269).

El lento declinar de la institución, sin embargo, empieza en ese mismo 1940, con la salida de su fundador John Andrew Rice. Theodore Dreier deja la escuela en 1949 y también Josef y Anni Albers se trasladan a la Universidad de Yale en ese mismo año. La única figura destacada que queda hasta el final es el poeta Charles Olson, que, en calidad de último rector, cierra la escuela por falta de fondos en 1956.

\section{El arte en tiempos de Gran Depresión: Dewey y el Federal Art Project}

El Federal Art Project (FAP) fue uno de los proyectos más ambiciosos llevados a cabo en el marco del New Deal durante la presidencia de Franklin D. Roosevelt (19331945). Inaugurado en 1935, era parte del más extenso Works Progress Administration (WPA), una agencia estatal que promovía la reinserción laboral en obras públicas de millones de trabajadores desempleados al tiempo de la Gran Depresión (1929-1939). La importancia del FAP para el desarrollo de un arte auténticamente estadounidense, así como su vinculación con el pensamiento pragmatista, son elementos que han sido subrayados, entre otros, por Aline Wiame:

En este período del New DeaI después de la crisis de 1929, la política artística de Estados Unidos está en la misima situación que las políticas económicas y sociales: se revisa de arriba a abajo. Sin duda hay razones patrióticas para ello: se trata de afirmar la singularidad de la escena artística estadounidense y las propuestas innovadoras de Dewey pueden ayudar a dibujar los contornos. Pero, y aquí es donde la contribución de Dewey es crucial, se intenta sobre todo desarrollar la dimensión social de las prácticas artísticas. El Federal Art Project afirma la importancia del arte ya que participa en la vida de la comunidad, ya que alienta al público en general a ser parte de la experiencia estética ${ }^{33}$.

El director y principal promotor del FAP fue Holger Cahill (nacido Sveinn Kristjan Bjarnarsson), un curador y crítico de arte de origen islandés. Criado en el Midwest en el seno de una familia pobre y pronto abandonado por ella, Cahill nunca recibió una educación regular hasta la adolescencia. Luego de trasladarse a Nueva York en los años ' 10 , empezó a tomar clases de periodismo y escritura creativa y se insertó con éxito en la vida de la boheme neoyorquina de aquella época. A principios de los años veinte se había convertido en una autoridad en la escena artística de Nueva York, siendo nombrado director de relaciones públicas de la Society of Independent Artists y obteniendo la conducción de un programa radial sobre arte.

En esa misma década comienza su colaboración con John Cotton $\mathbb{D a n a}$, director del Museo de Newark, que fue fundamental por dos motivos. En primer lugar, porque gracias a la amistad y protección de $\mathbb{D} a n a$, Cahill se convirtió en unos de los más destacados curadores de exposiciones de arte moderno y folklórico del país. En segundo lugar, porque Dana era un lector entusiasta de Dewey y fue determinante, junto con el pase de Cahill por la New School of Social Research y la Universidad de Columbia, para su ininiciación en el pragmatismo clásico ${ }^{34}$. Antes de asumir el cargo como director del

ser más que una frase (...). La democracia tiene que nacer de nuevo con cada nueva generación, y la educación es su partera" (MW, 9, p. 139).

${ }^{33}$ WIAME, Aline. Ob. Cit., p. 20.

${ }^{34}$ JEFFERS, Wendy. "Holger Cahill and American Art". En Archives of American Art Journal, 31, 4, 1991, pp. 2- 
FAP, en tanto, en 1932 Cahill fue nombrado director del prestigioso MoMa de Nueva York.

La admiración de Cahill por la filosofía de Dewey se hace evidente en los dos ejes principales de su "programa de acción": el abandono de una concepción elitista del arte, concebido como mero objeto de museo, por un lado y la eliminación de toda distinción entre bellas artes y artes aplicadas en favor de su plena integración en la experiencia cotidiana, por el otro. Sobre el primer punto, Cahill comparte las mismas inquietudes de Dewey acerca del origen y del rol social de los museos luego de la expansión del capitalismo. En New Horizons in American Art el crítico de origen islandés sostiene:

Luego de la Guerra Civil (...) [hubo] una rápida expansión de la industria para el dominio de grupos sociales que no tenían tradición de mecenazgo artístico y tampoco interés en el arte, sino como símbolo de la distinción social recientemente adquirida o como exposición de objetos llamativos (...). La nueva generación de coleccionistas exige lo grandioso, lo vulgar, lo espectacular, lo recargado, lo pretencioso (....). La arqueología y los mercantes más astutos los han provisionado con objetos que de algún modo habían sido identificados con la grandeza pasada y aún se identificaban con el gusto de las personas socialmente poderosas en Europa ${ }^{35}$.

Siguiendo las directivas de su ex profesor en la Universidad de Columbia, el proyecto de Cahill apuntaba a superar concretamente el dualismo entre arte y vida, producción artística y goce estético, artista y ciudadano. La medida más drástica del experimento impulsado por Cahill consistía en la superación del museo y de la galería como lugares exclusivos de exposición. En los 48 estados en donde actuó el FAP pinturas murales, afiches y esculturas ocupaban normalmente lugares públicos como muros, plazas y escuelas. Según la lectura de Masamichi Ueno,

el FAP provocó un cambio en la concepción social del arte a través de su influencia sobre artistas, críticos y educadores. Cuando los artistas producían sus trabajos, no tomaban conciencia solo de aficionados y coleccionistas, sino también de la gente común, ya que sus obras se encontraban y estaban expuestas públicamente en las comuinidades ${ }^{36}$.

Cahill comparte con Dewey la misma visión del arte no como un objeto acabado y útil al mero goce pasivo, sino como un proceso que involucra activamente el productor y el espectador. Más allá de ofrecer clases y talleres de arte gratuitos, los artistas del FAP a menudo trabajaban en sus obras a la vista del público, que así podía ser testigo del proceso de creación. Este argumento ha sido desarrollado por Jane De Hart Mathews en los siguientes términos:

En el proceso de creación [los espectadores] descubrían que el arte no era algo para ser guardado en instituciones exclusivas, sino "belleza para el uso" uso en "el más amplio sentido humano". Al enfatizar el proceso artístico y la respuesta pública, Cahill buscó una definición de arte que Dewey había proporcionado recién el año anterior. Al proporcionar acceso a lo que el filósofo llamó "el arte como experiencia", el New Deal podía crear la audiencia masiva - el sello distintivo de la democracia cultural ${ }^{37}$.

El segundo eje del programa de Cahill apuntaba a la eliminación de toda jerarquización entre bellas artes y artes útiles y tecnológicas, según una concepción que

11; TRASK, Jeffrey. Things American: Art Museums and Civic Culture in the Progressive Era. Filadelfia: University of Pennsylvania Press, 2012, pp. 98-104; 231-232.

${ }^{35}$ CAHILL, Holger. New Horizons in American Art. Nueva York: Museum of Modern Art, 1936, p. 11.

${ }^{36}$ UENO, Masamichi. Ob. Cit., p. 137.

${ }^{37}$ DE HART MATHEWS, Jane. "Arts and the People: The New Deal Quest for Cultural Democracy". The Joumal of American History, 62, 2, 1975, pp. 323. 
ya Dewey había claramente expuesto en su teoría estética ${ }^{38}$. La influencia de las bellas artes sobre las artes útiles tenía como objetivo el mejoramiento de las segundas y, en un sentido más amplio, un mejoramiento de la vida de las personas en la búsqueda de la que Dewey define la "Gran Comunidad" [Great Community] ${ }^{39}$.

Para Cahill, la producción industrial ha generado "un desorden terrible de cosas feas, y esto, a su vez, ha desembocado en una degradación del gusto popular, ya que estos objetos constituyen el único arte que mucha gente conoce ${ }^{240}$. Este llamado a la recuperación de la estética en los objetos que nos rodean en lo cotidiano es uno de los rasgos más evidentes en Art as Experience. Dewey, en particular, sostiene que "los utensilios domésticos útiles de la casa, mantos, esteras, jarros, platos, arcos, lanzas, eran decorados con tanto cuidado que ahora vamos en su búsqueda y les damos un lugar de honor en nuestros museos. Sin embargo, en su propio tiempo y lugar, tales cosas eran medios para exaltar los procesos de la vida cotidiana" ( $A E, p$. 7). En el mismo año en que publica Art as Experience Dewey es llamado al MoMa como jurado en una exposición de diseño industrial titulada "Machine Art". Luego de haber elegido una turbina de motor como el objeto más lindo, Dewey declara: "En mi opinión es una prueba convincente de que no hay una oposición esencial entre la producción por utilidad y la producción por belleza" ${ }^{m 41}$.

Uno de los artistas más beneficiados por el FAP fue Thomas Hart Benton, destacado exponente del realismo social estadounidense desde los años sucesivos a la Primera Guerra Mundial, conocido también por haber sido el maestro de Jackson Pollock. Es manifiesta su cercanía al pensamiento de Dewey, facilitada también por su experiencia, como el mismo Cahill, en la New School of Social Research:

Benton admitió su deuda con el filósofo norteamericano. Tenía conocimiento de Dewey desde 1928 y había leído más de su fillosofía que de Art as Experience. El énfasis que los escritos de Benton ponían en las emociones, la experiencia directa y el arte viviente muestran que de vez en cuiando tomaba literalimente ideas y conceptos claves de Dewey. Los contemporáneos de Benton notaron rápidamente la manera en que empezó a formular una terminología adaptada al idioma de Dewey. Jacob Burck, en Art Magazine, notó que Benton, siguiendo el ejemplo de Dewey, había empezado a hablar "de la función social de la pintura muiral" 42 .

Si Benton es probablemente el caso más conocido de artista del FAP inspirado por las teorías deweyanas, algunos de los futuros exponentes del Expresionismo Abstracto se beneficiaron igualmente del programa estatal. Entre ellos, se recuerdan el ya mencionado Jackson Pollock, Willem De Kooning y Arshile Gorky.

A pesar de la deuda evidente que Cahill y el FAP tenían con la filosofía de Dewey, el autor de Art as Experience nunca se involucró en el proyecto, más bien siempre manifestó su escepticismo hacia el New Deal de Roosevelt en general, y el FAP en particular ${ }^{43}$. En 1938 Dewey y Albert C. Barnes, juntos con el periodista Henry Hart, crearon en Filadelfia la institución Friends of Art and Education como vehículo oficial de sus vehementes protestas en contra de "las prácticas viciosas de la local sección del WPA Art Project" (BARNES en Correspondence II, 04395). El principal motivo de sus quejas era el manejo del FAP de Pensilvania por parte de la directora Mary Cullen, una ex docente de literatura y ex estudiante de la Barnes Foundation, cuya actividad de

\footnotetext{
${ }^{38}$ Sobre este punto véase $A E$, p. 256.

${ }^{39}$ En The Public and Its Problems (1927) Dewey subraya la centralidad del arte para el desarrollo de una Gran Comunidad [Great Community]. $L W, 2$, p. 351.

${ }^{40}$ CAHILL, Holger。Ob. Cit, 19.

${ }^{41}$ ROBINS, Alexander. Ob. Cit., p. 168.

${ }^{42}$ BUETTNER, Stewart. Ob. Cit., pp. 389-390. Sobre la cercanía de Benton con las teorías de Dewey ver también DEL CASTILLO, Ramón。Ob. Cit., pp. 85-86.

${ }^{43}$ Dos textos "menores" de 1933 y 1934 muestran claramente la posición crítica de Dewey sobre las políticas del New Deal: "New Deal Program Must Be Appraised" (LW, 9, pp. 281-28) e "Introduction to Challenge to the New DeaI" (LW, 9, pp. 297-299).
} 
promoción artística terminó contrastando con la de la Fundación dirigida por Barnes y Dewey ${ }^{44}$.

En tanto, las protestas de los sectores más conservadores del Congreso, por un lado (en 1939 se cerró primero al Federal Theatre Project acusado de subversión e inmoralidad) y el enorme esfuerzo bélico desde 1940, por el otro, provocaron un progresivo decaimiento del FAP hasta su fin oficial en 1943. Sin embargo, su influencia en el desarrollo sucesivo de las artes en Estados Unidos ya había sido decisiva.

Esta integración del arte y la vida, lograda mediante la integración de las artes bellas y de las artes prácticas, representó un nuevo movimiento en el arte estadounidense. Tal como lo había prescrito Dewey, Cahill había establecido una conciencia artística nacional. Lo que fue único de este experimento fue que no cambió el estilo del arte, ni forzó a los artistas a ajustarse a ningún estilo sugerido; la fuerza central del experimento fue entregada por la audiencia. La preocupación principal era contrarrestar la revolución industrial y ayudar a restablecer la sensibilidad requierida para reparair la brecha entre el artista y la sociedad ${ }^{45}$.

\section{John Dewey: pragmatismo y arte}

Tanto el título (giro estético y vanguardia) como el subtítulo (acerca de la influencia del pragmatismo de John Dewey en artistas norteamericanos) de este artículo intentan poner énfasis en dos ejes: en primer lugar, que existe un giro estético en la filosofía pragmatista de John Dewey a partir de la década del veinte; en segundo lugar, que dicho giro estético tiene una enorme influencia en la vanguardia artística norteamericana pero que sufre, en la historia del arte, un ostracismo similar al que padeció la filosofía de Dewey después de la Segunda Guerra Mundial. Desde un punto de vista histórico-filosófico, sin embargo, a partir de la década del ochenta tanto la filosofía de Dewey, en general, como su estética, en particular, han tenido un notable resurgimiento en la agenda actual como muestran los trabajos de Thomas M. Alexander, Mark Johnson, Casey Haskins y, especialmente, la somaestética de Richard Shusterman. A nuestro juicio, un resurgimiento similar tiene que ser llevado a cabo en la historia del arte que tendría que dar cuenta del impacto directo que tuvo la filosofía de Dewey en la escena artística norteamericana.

En este contexto surgen una serie de interrogantes. El primero: ¿en qué consistió, la influencia de Dewey en los artistas estadounidenses? Otro: ¿cuál es el vínculo entre su giro estético y la vanguardia? Finalmente: ¿por qué existe un ostracismo de la estética de Dewey en la historia del arte similar al que surge en la filosofía?

La respuesta a la primera pregunta debe necesariamente tomar en consideración el vínculo indisoluble que Dewey establece entre experiencia estética, educación y democracia (explicitado en Democracy and Education y otros escritos como "Art in Education-Education in Art" ( $L W, 2$, pp. 111-115) de 1926). Como se mostró en las secciones anteriores el vínculo entre estética y educación, tal como lo entiende Dewey, es central tanto para el BMC como para el FAP, instituciones que redefinieron el rol social del artista en Estados Unidos y prepararon el camino para la llegada de las neovanguardias de posguerra. En relación a este vínculo, particularmente en el $\mathrm{BMC}$, James M. Harding escribe:

\footnotetext{
${ }^{44}$ UENO, Masamichi, Ob. Cit., pp. 132-137. Véase también el ataque público de Dewey de 1938 en contra de Emanuel M. Benson, figura central del WPA - Federal Art Project "Report on "Forms of Art" Exhibition at the Philadelphia Museum of Art" (LW, 17, pp.128-129).

${ }^{45}$ MAVIGLIANO, George T. “The Federal Art Project: Holger Cahill Program of Action”. En Art Education, 37, 3, 1984 , p. 30.
} 
Independientemente de si Dewey lo reconoció, el Black Mountain College constituyó un nexo vivo de sus filosofías de educación y del arte, especialmente cuando ellas se solapan en una apelación enfática a un lugar alternativo de expresión y experiencia artística, un lugar que va conscientemente en contra de la corriente cultural principal ${ }^{46}$.

Con respecto a la segunda pregunta, es posible no solo rastrear un substrato cultural deweyano común a las distintas vanguardias estadounidenses (Expresionismo Abstracto, Action Painting, Happening, Fluxus, Minimalismo, Land Art, etc.), como han llevado a cabo admirablemente Buettner, Berube y Dreon, sino también demostrar que esta influencia es uno de los factores que determinaron una brecha entre las vanguardias europeas de principios del siglo veinte y las neovanguardias. En esa dirección, David Hopkins reconoce que, más allá de algunos elementos comunes, existe una gran diferencia entre los primeros movimientos europeos de vanguardia y los surgidos luego de la Segunda Guerra Mundial. En sus términos:

Existen muchas afinidades estructurales entre Dadá y Fluxus, o el Surrealismo y el Situacionismo. Pero, zes posible que el tono general de producción de gran parte del arte de vanguardia después de la Guerra era significativamente distinto del de comienzo de siglo? Una forma de caracterizar el arte vanguardista de los '50 y '60 es afirmar que sus operaciones eran simplemente menos ambiciosas, sus provocaciones y gestos menos altisonantes con respecto al comienzo del siglo veinte (...). John Cage es el típico ejemplo de vocero de las neovanguardias en términos de esta tendencia auto-desinflante ${ }^{47}$.

La respuesta más convincente a la cuestión planteada por Hopkins es la interpretación de las neovanguardias de James $\mathbb{T}$. Harding. En lugar de reconocer una actitud "auto-desinflante" en los artistas norteamericanos, el conocido historiador del teatro interpreta sus propuestas como alternativas constructivas al nihilismo de los europeos. En otras palabras, mientras los europeos a principios del siglo XX se hicieron promotores de una anti-cultura, es decir de una cultura que "elude la conciencia material a través de una reestructuración radical y completa del orden social", los norteamericanos encontraron en la propuesta de Dewey "un camino típicamente estadounidense", para construir una contracultura alternativa a la cultura dominante y capaz de "romper la lógica adoctrinada del capitalismo".

Sobre esta nueva actitud encarnada por John Cage Harding sostiene: "Mientras que el nihilismo radical de la Vanguardias europeas se inclinaba, aunque ineficazmente, hacia la negación a gran escala, (...) Cage, que practicaba su arte en ambientes fuertemente influenciados por la filosofía de Dewey, siguió un nuevo conjunto de estrategias" política que (...) ofrece una táctica eficaz para combatir la orden social" sin poner "el objetivo de su batalla en un futuro no especificado y que puede ser postergado indefinidamente". La diferencia principal entre Cage y los europeos, así como su actitud distinta, definida sin razón por Hopkins "auto-desinflante", se explica con que "la estética de Cage se aferra al presente en lugar de ir hacia el futuro" 49.

Esta estética de la afirmación, que no condena el pasado a priori y que construye el futuro con los materiales del presente, se refleja, otra vez, en el pensamiento de Dewey:

Para el ser plenamente vivo, el futuro no es ominoso, sino prometedor; rodea al presente como uin halo. Consiste en posibilidades que se sienten como una

${ }^{46}$ HARDING, James M. The Ghosts of the Avant-Garde(s): Exorcising Experimental Theatre and Performance. Ann Arbor: The University of Michigan Press, 2013, pp. 66-67.

${ }^{47}$ HOPKINS, David. "Art and Life... and Death: Marcel Duchamp, Robert Morris and the Neo-Vanguarde Irony". En HOPKINS, D. y SCHAFFNER, K.A., Neo-Avant-Garde. Amsterdam: Rodopi, 2006, p. 19.

${ }^{48}$ HARDING, James M., Ob. Cit., p. 69.

${ }^{49}$ Idem, Ibidem, p. 73. 
posesión de lo que está aquí y ahora. En la vida como tal, todo se solapa y se funde. Pero todos vivimos muy a menudo con aprensiones de lo que el futuro puede traer, y estamos divididos dentro de nosotros mismos. Aun cuando no estemos muy ansiosos, no disfrutamos el presente porque lo subordinamos a lo que está ausente. $\mathrm{A}$ causa de este frecuente abandono del presente por el pasado y el futuro, los periodos felices de una experiencia (...) llegan a constituirse en un ideal estético. Solamente cuando el pasado deja de perturbar y las anticipaciones del futuro no trastoman, el ser está enteramente unido con su ambiente y (...) plenamente vivo. El arte celebra con peculiar intensidad los momentos en que el pasado refuerza el presente y en los que el futuro es vin acelerador de lo que ahora es ( $A E_{,}$, p. 20. Cursiva nuestra).

Si se examinan algunos escritos de artistas de las vanguardias (a ambos lados del Océano), es posible recuperar la contraposición de la que habla Harding entre el nihilismo europeo y pragmatismo estadounidense. El dadaísta Hugo Ball, por ejemplo, escribe en 1927:

¿Qué significa todo esto? Tal vez una única cosa, que el mundo ha llegado a un punto muerto, se encuentra bajo el signo de la pausa general. Que ha amanecido un Viermes Santo universal, que, en este caso concreto, se percibe con más fuerza fuera de la Iglesia que en ella misma; que el calendario litúrgico se rompe y que Dios sigue muerto en la cruz el día de Pascua. Las famosas palabras del filósof́o, «Dios ha muerto», empiezan a tomar forma a nuestro alrededor ${ }^{50}$.

Cuarenta años después, en Estados Unidos, John Cage se expresa con un tono radicalmente distinto con respeto al artista alemán: "[Debemos] abrir nuestros ojos y oídos cada día viendo la vida como lo excelente que es (...). Aceptar lo que venga independientemente de las consecuencias, es no tener miedo o estar lleno de ese amor que proviene del sentido de unidad con todas las cosas"51. También Allan Kaprow, en una histórica entrevista con Susan Hapgood, se desliga parcialmente de las vanguardias europeas y reconoce, en cambio, las raíces específicamente norteamericanas y pragmatistas de su arte.

En la medida en que las personas comentaban sobre lo que estábamos haciendo en este particular evento o en otro lugar, hablaban sobre Dadá (....). A menudo pensaba que nos asociaban erróneamente con Dadá. El anti-arte no es algo que los dadaístas inventaron. Existe un hilo conductor de "Ia vida es mejor que el arte" que surge al menos en la época de Wordsworth y que, a través de Emerson y Whitman, llega hasta John Dewey y más allá, enfatizando el arte como experiencia, tratando de fusionar de nuevo arte y vida - esta es la tradición que me influenció mucho (Cursiva nuestra) ${ }^{52}$.

Con respecto a la tercer pregunta - por qué la influencia del pensamiento de Dewey ha sido subestimada en los estudios de historia del arte -la historiadora del arte Hannah Higgins, hija del artista de Fluxus Dick Higgins, apunta a la crítica

\footnotetext{
${ }^{50}$ BALL, Hugo. La huida del tiempo. Trad. de R. BRAVO DE LA VARGA. Barcelona, Acantilado, 2005 [1927], p. 209.

${ }^{51}$ El texto de Cage se encuentra en HIGGINS, Hannah. Fluxus Experience Berkeley y Los Ángeles: University of California Press, 2002, p. 83. Confróntense las ideas de Cage sobre "el sentido de unidad con todlas las cosas" con lo que Dewey escribe en Art as Experience "La vida institucional de la humanidad está marcada por la desorganización (...) y esta separación estática es aceptada como la esencia misma del orden (....). La vida se segmenta y los segmentos institucionalizados se clasifican en altos y bajos (...). Vemos sin sentir; oímos, pero solamente información de segunda mano, porque no está reforzado por la visión. Tocamos, pero el contacto permanece tangencial porque no se funde con las cualidades sensibles que están bajo la superficie. Usamos los sentidos para despertar la pasión, pero no para satisfacer el interés de la intuición, no porque ese interés no esté potencialmente presente en el ejercicio de los sentidos, sino porque cedemos a condiciones de vida que obligan a los sentidos a quedar como una excitación superficial ( $A F$, pp. 22-23).

52 Ver en HAPGOOD, Susan. Neo-Dada: Redefining Art, 1958-1962. Nueva York: American Foundation of Arts, 1994, p. 115.
} 
postestructuralista como a la principal responsable del ostracismo de la filosofía de Dewey. En una entrevista con Jeff Abell sostiene:

Dewey fue alguien que pasó de moda con el postestructuralismo, pero fue muy importante para muchos de los artistas de la década del sesenta, en el momento en que estaban haciendo su trabajo. Allan Kaprow leía a Dewey, hacía happenings y estudiaba con Cage. Daisetz Suzuki, cuando enseñaba en la Universidad de Columbia, tenía una foto de Dewey sobre su escritorio. Y Suzuki es crucial para Cage. Así que Dewey estaba en el centro de la forma en que estos artistas entendían su relación con los materiales. También formó parte del consejo del Black Mountain College, donde Cage y Merce Cuniningham enseñaron y se convirtieron en una especie de abuelos de Fluxus. Muchas de las personas que estaban experimentando con materiales en la manera de los '60 -esa manera sensual en la que estoy interesada- tenían una conexión con Dewey, aunque esta fue completamente oscurecida cuando los desafíos que este trabajo trae al discurso verbal se volvieron explotados por el postestructuralismo y el discurso político. Por tanto, Dewey es tratado como si fuera una especie de idea loca en la que estaban los artistas, y luego llega ese globo de teoría postestructuralista que nos dice lo que realmente estaban haciendo los artistas ${ }^{53}$.

\section{Conclusiones}

En las páginas iniciales se sostuvo que el propósito del presente artículo era recortar, reconstruir y analizar algunas características fundamentales de la filosofía de Dewey que más han influido en el desarrollo de dos instituciones creadas entre las dos Guerras Mundiales: el Black Mountain College y el Federal Art Project Para llevar adelante esta tarea, en la primera sección se presentaron algunas características centrales de su pensamiento. En la segunda y tercera sección, en tanto, se sostuvo que la influencia de Dewey (tanto en el BMC como en el FAP) se debe principalmente a que ambas instituciones conciben la vinculación entre educación y estética bajo la inspiración del gran pragmatista clásico. En la cuarta sección del trabajo se mostró que la influencia del pragmatismo de Dewey en la vanguardia norteamericana es decisiva para que ésta se conciba como contracultural y no como anticultural (tal como lo hace la vanguardia europea de principios del siglo veinte). Esa influencia, sin embargo, no es debidamente considerada en la literatura de historia del arte, lo que permite inferir que al bien conocido ostracismo que sufre el pensamiento de Dewey en el ámbito filosófico después de la Segunda Guerra Mundial (que tiene su origen principal en la hegemonía de la filosofía analítica) existe un fenómeno similar en la historia del arte. En otros términos: el inadecuado desinterés por la estética de Dewey en la historia del arte (debido a la preeminencia de las teorías postestructuralistas que por diversos motivos son refractarias al pragmatismo en general) ha invisibilizado la decisiva influencia de Dewey en las neovanguardias. En consecuencia, en relación a la estética de Dewey es

\footnotetext{
${ }^{53}$ HIGGINS, Hannah y ABELL, Jeff. "Hannah Higgins Interviewed by Jeff Abell". En Mouth to Mouth, Winter 2004, http://mouthtomouthmag.com/higgins.html. (Úlímo acceso: 03/02/ 2019). En la misma dirección apunta Jacob: "A medida que la atención se trasladó hacia los teóricos marxistas y posmodemos, Dewey fue dejado atrás. Una posible explicación de por qué no es uno de los teóricos preeminentes puede ser que, simplemente, nunca separó la teoría de la práctica. Buscar eso en Dewey es malinterpretar la naturaleza real de la experiencia" (JACOB, Mary J., Ob. Cit, p. 3). Una primera aproximación al tema de la relación entre Dewey y el arte estadunidense posterior a la Segunda Guerra Mundial se encuentra en un artículo de Leon Jacobson, publicado en 1960 en plena época de neovanguardias. El autor afirma que existe una incongruencia entre la estética "objetiva" de Dewey y las prácticas artísticas "no-objetivas" que se estaban desarrollando en aquella época. Considera por tanto Art as Experience como un texto irrelevante para el desarrollo del arte contemporáneo. JACOBSON, Leon. "Art as Experience and American Visual Art Today". En The Joumal of Aesthetics and Art Criticism, 19, 2, pp. 117-126. Alexander Robins, en una conversación con los autores del presente artículo, ha lamentado, al igual que Higgins y Jacob, la persistencia de posiciones similares a la de Jacobson tanto entre los críticos estadounidenses como entre los historiadores del arte. Sin embargo, el reciente libro de Mary J. Jacob, direccionado principalmente a artistas, curadores y educadores, puede ayudar a superar esta tendencia aún hegemónica.
} 
preciso realizar en la historia del arte una tarea de rehabilitación similar a la que la filosofía llevó a cabo (con Rorty, Shusterman y Alexander, entre otros) a partir de la décadla del ochenta.

\section{Referências}

ADAMIC, Louis. "Education on a Mountain: The Story of Black Mountain College". Harper's Monthly Magazine, 172 (1936). 516-530.

ALBERS, Josef. "Art as Experience". Progressive Education 12.6 (1935). 391-393.

"A Note on the Arts in Education". American Magazine of Art 29.4 (1936). 233.

ALEXANDER, Thomas M. John Dewey's Theory of Art, Experience and Nature: The Horizons of Feeling. Albany: Suny Press, 1987.

BALL, Hugo. La huida del tiempo. Trad. de Roberto Bravo de la Varga. Barcelona, Acantilado, 2005 [1927].

BARBEREAU, Yoann. "Expérience et performance. Fragments d'un dialogue pragmatiste". Revue d'Esthétique 44. (2003). 25-36.

BEARDSLEY, Monroe. Aesthetics from Classical Greece to the Present: A Short History. Nueva York: The Macmillan Co., 1966.

BERUBE, Maurice R. "John Dewey and the Abstract Expressionists". Educational Theory 48 (1998). 211-227.

BUETTNER, Stewart. "John Dewey and the Visual Arts in America". Journal of Aesthetics and Art Criticism 33 (1975). 383-391.

CAHILL, Holger. New Horizons in American Art. Nueva York: Museum of Modern Art, 1936.

CROCE, Benedetto. "Intorno all'estetica del Dewey". La Critica 38 (1940). 348-353.

DE HART MATHEWS, Jane. "Arts and the People: The New Deal Quest for Cultural Democracy". The Journal of American History 62.2 (1975). 316-339.

DEL CASTILLO, Ramon. "La corrosión de la experiencia. Populismo, abstracción y cultura de masas. En ARENAS, L., FAERNA, M。, DEL CASTILLO, R. (eds.), John Dewey. Una estética de este mundo. Zaragoza: Prensas de la Universidad de Zaragoza, 2018. 77 96.

DEWEY, John. Democracy and Education. The Collected Works of John Dewey. Middle Works, 9. J.A. Boydston (ed.). Carbondale y Edwardsville: Southern Illinois University Press, 1980 [1916].

----. "Individuality as Experience". The Collected Works of John Dewey. Later Works, 1. J.A. Boydston (ed.). Carbondale y Edwardsville: Southern Illinois University Press, 1983 [1925]: $1-6$.

---- "Affective Thought". The Collected Works of John Dewey. Later Works, 1. J.A. Boydston (ed.). Carbondale y Edwardsville: Southern Illinois University Press, 1983 [1925]: 3-9.

----. "Art in Education-Education in Art". The Collected Works of John Dewey. Later Works, 2. J.A. Boydston (ed.). Carbondale y Edwardsville: Southern Illinois University Press, 1984 [1926]: 111-115.

---- The Public and Its Problems. The Collected Works of John Dewey. Later Works, 2. J.A. Boydston (ed.). Carbondale y Edwardsville: Southern Illinois University Press, 1984 
[1927].

---- "What Is It All About?". The Collected Works of John Dewey. Later Works, 6. J.A. Boydston (ed.). Carbondale y Edwardsville: Southern Illinois University Press, 1985 [1931]: 330-334.

---- "New Deal Program Must Be Appraised". The Collected Works of Jolhn Dewey. Later Works, 9. J.A. Boydston (ed.). Carbondale y Edwardsville: Southern Illinois University Press, 1986 [1933]: 281-282.

---- "Introduction to Challenge to the New Deal". The Collected Works of John Dewey. Later Works, 9. J.A. Boydston (ed.). Carbondale y Edwardsville: Southern Illinois University Press, 1986 [1934]: 297-299.

---. El arte como experiencia. J. Claramonte (trad.). Barcelona: Paidos, 2008 [1934].

DÍAZ, Eva. The Experimenters: Chance and Design at Black Mountain College. Chicago y Londres: University of Chicago Press, 2015.

DREON, Roberta. Fuori dalla torre d'avorio: l'estetica inclusiva di John Dewey oggi. Génova y Milán: Marietti, 2012.

"Was Art as Experience Socially Effective? Dewey, the Federal Art Project and Abstract Expressionism". The European Journal of Pragmatism 5 (2013). 279-292.

FISHER, Jonathan. "The Life and Work of the College: Towards the History of Black Mountain College as an Institution of Progressive Higher Education". Tesis para optar al grado de Magíster en Educación. Universidad de British Columbia, 2013.

FÜSSL, Karl-Heinz. "Pestalozzi in Dewey's Realm? Baulhaus Master Josef Albers among the German- speaking Emigrés' Colony at Black Mountain College (1933-1949)"。 Pedagogica Historica 42.1-2 (2006). 77-92.

HAPGOOD, Susan. Neo-Dada: Redefining Art, 1958-1962. Nueva York: American Foundation of Arts, 1994.

HARDING, James M. The Ghosts of the Avant-Garde(s): Exorcising Experimental Theatre and Performance. Ann Arbor: The University of Michigan Press, 2013.

HICKMAN, L. (ed.). The Correspondence of John Dewey. Vols. II y III. Charlottesville: Intelex, 2008.

HIGGINS, Hannah y ABELL, Jeff. "Hannah Higgins Interviewed by Jeff Abell". Mouth to Mouth, Winter 2004, http://mouthtomouthmag.com/higgins.html. Web. Último acceso: 03 de febrero de 2019 .

HIGGINS, Hannah. Fluxus Experience. Berkeley y Los Angeles: University of California Press, 2002.

HOPKINS, David. "Art and. Life... and Death: Marcel Duchamp, Robert Morris and the Neo-Vanguarde Irony". Neo-Avant-Garde. Amsterdam: Rodopi, 2006: 19-36.

JACOB, Mary Jane. Dewey for Artists. Chicago y Londres: The University of Chicago Press, 2018.

JAY, Martin. "Somaesthetics and Democracy: Dewey and Contemporary Body Art". Journal of Aesthetic Education 36 (2002). 55-69.

JEFFERS, Wendy. "Holger Cahill and American Art". Archives of American Art Journal 31.4 (1991). 2-11.

KATGENS-CRAIG, Margaret. The Bauhaus and America: First Contacts (1919-1936). Cambridge y Londres: MIT Press, 1999.

MAVIGLIANO, George T. "The Federal Art Project: Holger Cahill Program of Action". Art 
Education 37.3 (1984). 26-30.

MUMFORD, Lewis. The Golden Day: A Study in American Experience and Culture. Nueva York: Horace Liveright, 1926.

PEPPER, Stephen. "Some Questions on Dewey's Aesthetics". The Philosophy of John Dewey. Evanston: Northwestern University, 1939. 369-390.

PERROW, Charles. "Drinking Deep at Black Mountain College". Southern Cultures 19.4 (2013). 76-94.

PIRÓN, François. "Se rire de l'échec: le pragmatisme de George Maciunas". Revue Initiales 1 (2009). 27-32.

REYNOLDS, Katherine C. "Progressive Ideals and Experimental Higher Education: The Example of John Dewey and Black Mountain College". Education and Culture 14.1 (1997).1-9.

ROBINS, Alexander. "Aesthetics Experience and Art Appreciation: A Pragmatic Account". Tesis para optar al grado de Doctor en Filosofía. Universidad de Emory, Atlanta, 2015.

SENALDI, Marco. "Art as Experience e l'arte contemporanea". Esperienza estetica a partire da John Dewey. Palermo: Centro Internazionale Studi di Estetica, 2007. 49-60.

TRASK, Jeffrey. Things American: Art Museums and Civic Culture in the Progressive Era. Filadelfia: University of Pennsylvania Press, 2012.

UENO, Masamichi. Democratic Education and the Public Sphere: Towards John Dewey's Theory of Aesthetic Experience. Nueva York: Routledge, 2016.

WESTBROOK, Robert B. John Dewey and American Democracy. Ithaca y Londres: Cornell University Press, 1991.

WIAME, Aline. "L'art comme expérience et la pragmatique du spectateur: entre performance et philosophie". Tangence 108 (2015). 13-27. 